\title{
A Qualidade das Águas de Mesa Portuguesas
}

\author{
VICTOR M. M. LOBO* e MANUELA ARAÚJO**
}

\begin{abstract}
Efectuaram-se análises químicas dos parâmetros fundamentais em todas as águas de mesa que conseguimos obter no mercado nacional (todas portuguesas). Verifica-se que a maioria dos rótulos refere resultados de análise para poucos parâmetros. Contudo, de um modo geral, os valores indicados nos rótulos coincidem razoavelmente com as nossas análises e, quer estes quer os outros parâmetros analisados, mostram tratar-se de águas de boa qualidade segundo os critérios cientificamente aceites e também segundo a legislação em vigor.
\end{abstract}

\section{INTRODUÇ̃̃o}

Felizmente, o nosso pais tem grandes capacidades de produção de águas naturais de excelente qualidade para consumo humano, dadas as nossas condições orográficas e pluviais. Dai o mercado nacional ter uma abundante diversidade de águas de mesa (Tabela 1), sobretudo quando em comparação com o mercado britânico, americano ou australiano.

Infelizmente, a situação no que respeita a águas fornecidas por entidades municipais (ou similares) para distribuição domiciliária, em princípio também para consumo humano, já deixa muito a desejar, como os recentes acontecimentos na cidade de Évora relativamente ao teor em alumínio bem demonstram. 0 Decreto-Lei 74/90 de 7 de Março indica parâmetros químicos a ter em conta para a classificação de águas de abastecimento para consumo humano.

Para o presente trabalho adquirimos amostras de todas as águas que conseguimos encontrar em mercados de Coimbra, Porto, Vila Nova de Famalicão, etc., tendo colectado o conjunto descrito no mercado por nos parecer que, para 0 objectivo em causa, tal não se justificaria.

Procedemos a análises químicas destas e comparamos os valores por nós obtidos com os indicados nos rótulos, bem como com os critérios de qualidade estabelecidos pelo Decreto-Lei 74/90, muito embora este Decreto tenha em vista só águas de abastecimento para consumo humano.

\section{PARTE EXPERIMENTAL E CONCLUSÕES}

As amostras foram recolhidas dos seus locais de venda tal como se encontram, sendo nossa preocupação que estivessem devidamente seladas. Adquiriram-se geralmente 3 a 5 garrafas de 1.5 litros, ou 1 garrafão de 5 ।.

Foram analisados os parâmetros químicos indicados na Tabela 1 por métodos descritos na bibliografia desta área, nomeadamente [1] e [2] que, face a sua divulgação, nos dispensamos de descrever. Sao também já bem conhecidos os critérios de cariz médico e estatístico que levam aos conceitos de "Valor Máximo Recomendado" (VMR) e "Valor Máximo Admissivel" (VMA). Como e óbvio, será desejável que uma água não tenha, para um dado parâmetro, um valor superior ao VMR, e para poder ser considerada água de consumo, não pode ter um valor superior ao VMA. Note-se que há aspectos que se podem prender com condições logísticas relativas à distribuição da água, e não tanto à saúde humana. Assim, por exemplo, a recomendação segundo a qual o pH da água se não deve situar abaixo de 6.5 . prende-se com os problemas de corrosão (que por sua vez podem causar problemas de saúde) nas condutas de ferro galvanizado, normalmente usadas nos sistemas municipais. Assim, uma água de mesa como, por exemplo, "Água de Luso", que tem um $\mathrm{pH}=5.6$, embora abaixo do previsto para "águas de abastecimento para consumo humano", não deve obviamente ser rejeitada por esse facto como água de mesa [3].

A Tabela 1 apresenta, com 0 título "valor obtido", os resultados das análises por nos efectuadas para os seguintes parâmetros: pH, resíduo seco, condutibilidade, dureza total, alcalinidade total, hidrogenocarbonato (bicarbonato), oxidabilidade, sílica, cloreto, sulfato, nitrato, nitrito, cálcio, magnésio, ferro total e amónio. Mostra também, com o título "valor indicado", os valores fornecidos nos rótulos. Note-se que para muitos parâmetros os rótulos nada indicam (como, por exemplo, acerca dos nitratos).

As linhas desvio [(obt.-ind.)/med.] indicam, em termos percentuais, 0 desvio entre os valores das nossas análises e 0 indicado no rótulo (quando tal acontece), relativamente a média desses dois valores. Refira-se que, de um modo geral (salvo no caso de valores absolutos muito baixos), os desvios percentuais entre os valores indicados e os valores obtidos são relativamente baixos, tendo em consideração 0 intervalo de tempo decorrido entre as análises indicadas nos rótulos e as nossas análises. Exceptua-se o caso da água do "Caramulo" que, apesar de um pequeno desvio no resíduo seco, apresenta grandes desvios a nivel de composição, nomeadamente no $\mathrm{pH}$ hidrogenocarbonato, silica e cloreto.

Na generalidade, a composição das águas de mesa estudadas esta dentro dos limites indicados no D.L. 74/90, excepto para os seguintes casos (unidades tal como indicadas na Tabela 1; VMR = Valor Máximo Recomendado):

\section{Água Viva:}

condutibilidade $=444 ;$ VMR $=400$

cloretos $=61.5 ;$ VMR $=25$

sulfatos $=66.9 ;$ VMR $=25$

S. Lourenço:

cloretos $=34.1 ; \mathrm{VMR}=25$

Vimeiro:

condutibilidade $=1387 ;$ VMR $=400$

cloretos $=224.8 ; \mathrm{VMR}=25$

sulfatos $=92.9 ; \mathrm{VMR}=25$

cálcio $=125.5 ; \mathrm{VMR}=100$

amónio $=0.076 ; \mathrm{VMR}=0.05$

Pizões:

condutibilidade $=803 ;$ VMR $=400$

cloretos $=93.6 ;$ VMR $=25$

cálcio $=113.8 ;$ VMR $=100$

Note-se, contudo, que em nenhum destes casos se excede 0 valor máximo admissivel (VMA). 


\begin{tabular}{|c|c|c|c|c|c|c|c|c|c|c|c|c|c|c|c|c|c|c|}
\hline Água & & Fastio & Luso & Alardo & $\begin{array}{l}\text { Serra da } \\
\text { Estrela }\end{array}$ & Marăo & $\begin{array}{l}\text { Agua } \\
\text { Viva } \\
\end{array}$ & Cruzeiro & \begin{tabular}{|l|} 
São \\
Lourrenço
\end{tabular} & Vitalis & Caramulo & $\begin{array}{l}\text { Carva- } \\
\text { lhelhos }\end{array}$ & Serrana & \begin{tabular}{|l|} 
Săo \\
Silvestre
\end{tabular} & Vimeiro & Pizões & $\begin{array}{l}\text { Ladeira } \\
\text { Envendos }\end{array}$ & Grichöes \\
\hline pH (valor indicado) & Sorensen & 5.9 & 5.6 & 5.9 & 5.82 & 5.7 & 6.76 & 6.88 & \begin{tabular}{|l|}
6.6 \\
\end{tabular} & 5.3 & 5.5 & 7.91 & 5.9 & 7.1 & 7.00 & 7.2 & 5.0 & 5.3 \\
\hline $\mathrm{pH}$ (valor obtido) & Sorensen & 6.30 & 5.87 & 6.14 & 6.17 & 6.27 & 7.42 & 7.32 & 7.14 & 5.99 & 7.02 & 8.05 & 6.22 & 7.74 & 7.34 & 7.72 & 5.53 & 6.03 \\
\hline Deswo pH [(obt-Ind. .7$]]$ & & $5.7 \%$ & $3.9 \%$ & $3.4 \%$ & $5.0 \%$ & $8.1 \%$ & $9.4 \%$ & $6.3 \%$ & $7.7 \%$ & $9.9 \%$ & $21.7 \%$ & $2.0 \%$ & $4.6 \%$ & $9.1 \%$ & $4.9 \%$ & $7.4 \%$ & $76 \%$ & $10.4 \%$ \\
\hline Reaiduo Seco (valor indicado) & $m g 1$ & 34.2 & 40.0 & 28.0 & 26.0 & 27.3 & 288.6 & 146.6 & 206.0 & 46.6 & 85.4 & 245.5 & 37.6 & 180.6 & 917.0 & 530.0 & 28.0 & 32.8 \\
\hline Residuo Seco (valor obtido) & $m g t$ & 36.6 & 37.6 & 23.8 & 20.2 & 27.0 & 305.6 & 134.4 & 233.6 & 43.2 & 90.2 & 185.4 & 49.2 & 182.6 & 889.8 & 519.2 & 35.4 & 43.8 \\
\hline Desuo Res.Seco [(obt-Ind.).med.] & & $6.8 \%$ & $-6.2 \%$ & $-16.2 \%$ & $-25.1 \%$ & $-1.1 \%$ & $5.7 \%$ & $-8.7 \%$ & $12.6 \%$ & $-7.6 \%$ & $5.5 \%$ & $-27.9 \%$ & $26.7 \%$ & $1.1 \%$ & $-3.0 \%$ & $-2.1 \%$ & $23.3 \%$ & $28.7 \%$ \\
\hline Condutibilidade (valor obtido) & $\mu \mathrm{S} / \mathrm{tm}$ & 37.2 & 49.5 & 23.6 & 22.9 & 33.0 & 444 & 234 & 369 & 42.6 & 91.5 & 195 & 66.9 & 266 & 1387 & 803 & 31.4 & 47.3 \\
\hline Dureza Total (valor indicado) & $\mathrm{mg}$ сасози & & & & & & & 100 & & & & & & & & & & \\
\hline Dureza Total (valor obtido) & $m g$ СасО 3 & 7.5 & 9.4 & 3.8 & $4.7^{\circ}$ & 10.6 & 147 & 100 & 155 & 4.7 & 20.1 & 19.3 & 17.6 & 85.7 & 414 & 393 & 3.9 & 9.5 \\
\hline Alcalinidade Total (valor obtido) & $m g$ СасО 3 & 17.9 & 16.2 & 13.1 & 13.0 & 18.0 & 145 & 198 & 255 & 11.6 & 61.5 & 212 & 27.5 & 211 & 716 & 630 & 2.0 & 11.0 \\
\hline Bicarbonato (valor indicado) & mg НСОЗ & 9.2 & 11.0 & 6.6 & & 9.0 & & 126.3 & 134.2 & 7.3 & 14.6 & 133.8 & 8.6 & 114.7 & 441.6 & 383.1 & 1.7 & 2.4 \\
\hline Bicarbonato (valor obtido) & $\mathrm{mg} \mathrm{HCO3M}$ & 10.9 & 9.9 & 8.0 & 8.0 & 11.0 & 88.5 & 120.6 & 155.3 & 7.1 & 37.5 & 129.4 & 16.8 & 129.0 & 436.8 & 384.3 & 1.2 & 6.7 \\
\hline Deswo Bicarb. [(obt-ind.).ymed.] & & $17.1 \%$ & $-10.7 \%$ & $19.1 \%$ & & $19.8 \%$ & & $-4.6 \%$ & $14.6 \%$ & $-3.1 \%$ & $87.9 \%$ & $-3.3 \%$ & $64.4 \%$ & $11.7 \%$ & $-1.1 \%$ & $0.3 \%$ & $-32.9 \%$ & $946 \%$ \\
\hline Oxidabilidade (valor obtido) & $\mathrm{mg} O 21$ & 0.10 & 0.18 & 0.18 & 0.18 & 0.20 & 0.39 & 0.12 & 0.12 & 0.12 & 0.16 & 0.12 & 0.16 & 0.34 & 0.72 & 0.42 & 0.13 & 0.18 \\
\hline Silica (valor indicado) & $m g$ SiO2A & 13.1 & 13.0 & 12 & & 5.9 & & 13.6 & & 21.6 & 13.4 & 39.1 & 10.6 & & & & 10.9 & 7.2 \\
\hline Silica Mollbd. (valor obtido) & mg SiO2A & 14.1 & 13.2 & 13.3 & 11.1 & 7.1 & 33.0 & 14.5 & 14.8 & 20.0 & 38.5 & 41.0 & 13.4 & 14.1 & 13.0 & 25.0 & 9.2 & 7.7 \\
\hline 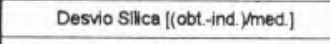 & & $7.4 \%$ & $1.5 \%$ & $10.3 \%$ & & $18.5 \%$ & & $6.4 \%$ & & $-7.7 \%$ & $96.7 \%$ & $4.7 \%$ & $23.3 \%$ & & & & $-16.9 \%$ & $6.7 \%$ \\
\hline Cloreto (valor indicado) & $m g c u$ & 4.6 & 8.5 & 2.4 & & 4.5 & & 16.5 & 38.3 & 7.4 & 23.8 & & & 34.1 & 221.9 & 103.7 & 6.8 & 9.2 \\
\hline Cloreto (valor obtido) & $\mathrm{mg} \mathrm{Cn}$ & 4.9 & 7.9 & 1.9 & 2.1 & 3.7 & 61.5 & 16.9 & 34.1 & 7.1 & 7.1 & 3.8 & 9.0 & 32.8 & 224.8 & 93.6 & 6.1 & 9.1 \\
\hline Desvio Cloreto [(obt-ind.)med.] & & $6.3 \%$ & $-7.3 \%$ & $-23.3 \%$ & & $-19.5 \%$ & & $2.4 \%$ & $-11.6 \%$ & $-4.1 \%$ & $-108.1 \%$ & & & $-3.9 \%$ & $1.3 \%$ & $-10.2 \%$ & $-10.9 \%$ & $-1.1 \%$ \\
\hline Sulfato (valor indicado) & mg so4n & & 1.1 & & & & & 1.6 & & 1.9 & 11.0 & & & & & 17.8 & & \\
\hline Sulfato (valor obtido) & $m g$ sO4n & 0.2 & 1.2 & 0.1 & 0.3 & 0.3 & 66.9 & 1.4 & 10.8 & 2.1 & 6.6 & 8.0 & 4.5 & 4.8 & 92.9 & 20.4 & 1.7 & 0.5 \\
\hline Desvio Sulfato [(obt-ind, .)med.] & & & $8.7 \%$ & & & & & $-13.3 \%$ & & $10.0 \%$ & $-50.0 \%$ & & & & & $13.6 \%$ & & \\
\hline Nitrato (valor indicado) & mg NO3A & & & 1.6 & & & & & & & & & & & & & & \\
\hline Nitrato (valor obtido) & mg NO3M & 2.5 & 1.7 & 2.0 & 1.4 & 1.5 & $<1.0$ & 0.8 & 12.1 & 1.9 & $<1.0$ & $<1.0$ & 2.5 & $<1.0$ & 6.3 & 19.9 & $<1.0$ & 4.0 \\
\hline Nitrito (valor obtido) & mg NO2A & 0.001 & 0.001 & 0.002 & 0.002 & 0.002 & $<0.001$ & 0.003 & 0.004 & $<0.001$ & $<0.001$ & $<0.001$ & 0.001 & 0.002 & 0.003 & 0.001 & 0.003 & $<0.001$ \\
\hline Sódio (valor indicado) & $m g ~ N a / ~$ & 5.0 & 6.9 & 3.5 & & 3.0 & & 10.2 & 20.2 & 6.0 & 20.7 & 49.6 & 6.5 & 31.1 & 157.7 & 34.0 & 3.9 & 6.2 \\
\hline Potassio (valor indicado) & $\mathrm{mg} K \mathrm{~K}$ & 0.6 & 0.8 & & & & & 5.8 & & 2.1 & 1.1 & & & & 23.2 & & & 0.85 \\
\hline Cálcio (valor indicado) & $\mathrm{mg}$ cal & 1.8 & 0.8 & 1.1 & & 2.2 & & 19.6 & 48.2 & 1.2 & 3.2 & & & 23.2 & 116.0 & 118.0 & 1.1 & 1 \\
\hline Calcio (valor obtido) & $\mathrm{mg} \mathrm{Cal}$ & 2.1 & 2.1 & 1.5 & 1.4 & 3.0 & 33.6 & 20.6 & 54.4 & 1.1 & 5.7 & 6.9 & 4.1 & 29.4 & 125.5 & 113.9 & 0.6 & 2.2 \\
\hline Desvio Calcio [(obt-ind. ./med.] & & $15.4 \%$ & $89.7 \%$ & $30.8 \%$ & & $30.8 \%$ & & $5.0 \%$ & $12.1 \%$ & $-8.7 \%$ & $56.2 \%$ & & & $23.6 \%$ & $7.9 \%$ & $-3.5 \%$ & $-58.8 \%$ & $75.0 \%$ \\
\hline Magnésio (valor indicado) & $m g \mathrm{Mgh}$ & & 1.6 & & & & & 12.4 & & & 1.2 & & & & & 29.2 & & \\
\hline Magnesio (valor obtido) & $m g$ Mgn & 0.5 & 1.0 & $<0.1$ & 0.3 & 0.8 & 14.9 & 11.9 & 4.6 & 0.5 & 1.4 & 0.5 & 1.8 & 3.0 & 24.4 & 26.4 & 0.6 & 1.0 \\
\hline Desvio Magnes. [(oot-ind.).Mmed] & & & & & & & & $-4.1 \%$ & & & $15.4 \%$ & & & & & $-10.1 \%$ & & \\
\hline Ferro Total (valor indicado) & $\mathrm{mg}$ Fen & & & & & $<0.05$ & & & & & 0.021 & & & & & & & \\
\hline Ferro Total (valor obtido) & $m g$ Fen & 0.011 & 0.003 & 0.011 & 0.001 & 0.011 & 0.033 & 0.032 & 0.025 & 0.017 & 0.021 & 0.012 & 0.004 & 0.011 & 0.019 & 0.014 & 0.018 & 0.019 \\
\hline Amónio (valor indicado) & mg NH4A & & & & & & & & & & 0.004 & & & & & & & \\
\hline Amónio (valor obtido) & $\mathrm{mg} \mathrm{NH4A}$ & 0.025 & 0.020 & 0.020 & 0.023 & 0.033 & 0.013 & 0.026 & 0.022 & 0.023 & 0.012 & 0.022 & 0.008 & 0.009 & 0.076 & 0.002 & 0.017 & 0.005 \\
\hline Fluoreto (valor indicado) & $m g F A$ & 0.2 & $<0.05$ & & & & & 0.15 & & & & 3.1 & & & & & & \\
\hline
\end{tabular}

Tabela 1 - Comparação entre os valores indicados nos rótulos das embalagens das águas de mesa com os valores obtidos das nossas análises. 
Nas figuras 1 a 7 apresentam-se, em gráfico de barras, comparações entre os valores obtidos nas nossas análises e os valores indicados nos rótulos para, respectivamente, os seguintes parâmetros: pH, resíduo seco, silica, hidrogenocarbonato, cloreto, sulfato e cálcio.

Da Fig. 1 verifica-se que todos os valores de $\mathrm{pH}$ medidos estão acima dos indicados nos rótulos, o que leva a crer tratar-se de uma consequência natural do armazenamento. Apenas a água do "Caramulo" apresenta uma grande diferença (de 5.5 para 7.0 ). As eventuais diferenças de temperatura de análise (de 17 a $25^{\circ} \mathrm{C}$ ) praticamente só se fazem sentir na casa dos centésimos. Como já foi dito, o VmA (valor mínimo admissível) no D.L. 74/90 (6.5) não e aqui aplicável.

Na Fig. 2 verificamos que o residuo seco determinado é muito próximo do indicado para todas as águas analisadas. Pode concluir-se que, de um modo geral, são águas pouco mineralizadas (10 águas com menos de $100 \mathrm{mg} / \mathrm{l}, 5$ águas entre 100 e $300 \mathrm{mg} / \mathrm{l}$ ); exceptuam-se as águas do "Vimeiro" $(\approx 900$ $\mathrm{mg} / \mathrm{l}$ ) e de "Pizões" ( $\approx 500 \mathrm{mg} / \mathrm{l})$. Todas as águas estão conforme 0 D.L. 74/90, que apresenta um VMA de $1500 \mathrm{mg} / \mathrm{l}$, não mencionando um VMR, e também obedecem ao valor limite recomendado pela Organização Mundial de Saúde de $1000 \mathrm{mg} / \mathrm{l}$. Geralmente uma água com mineralização inferior a $600 \mathrm{mg} / \mathrm{l}$ é considerada "boa", e "desagradável" para cima de $1200 \mathrm{mg} / \mathrm{l}$ [2].

Da Fig. 3 conclui-se que todos os valores obtidos para a sílica coincidem razoavelmente com os indicados nos rótulos (das que 0 indicam), salvo o caso da água do "Caramulo", cujo valor obtido e mais do dobro do valor indicado.

Também na Fig. 4 se verifica que os teores em hidrogenocarbonato são muito semelhantes aos indicados para todas as águas, salvo no caso da água do "Caramulo" em que, apesar da diferença em valor absoluto ser pequena por ter baixa concentração, o valor obtido é cerca do dobro do valor indicado.

Da Fig. 5 verifica-se que também a concentração obtida em cloretos está muito próxima dos valores indicados, com excepção da água do "Caramulo" que, neste caso, tem menos de metade da concentração indicada. 0 VMR de $25 \mathrm{mg} \mathrm{Cl} / /$ é ultrapassado pelas águas mais mineralizadas "Água Viva", "S.

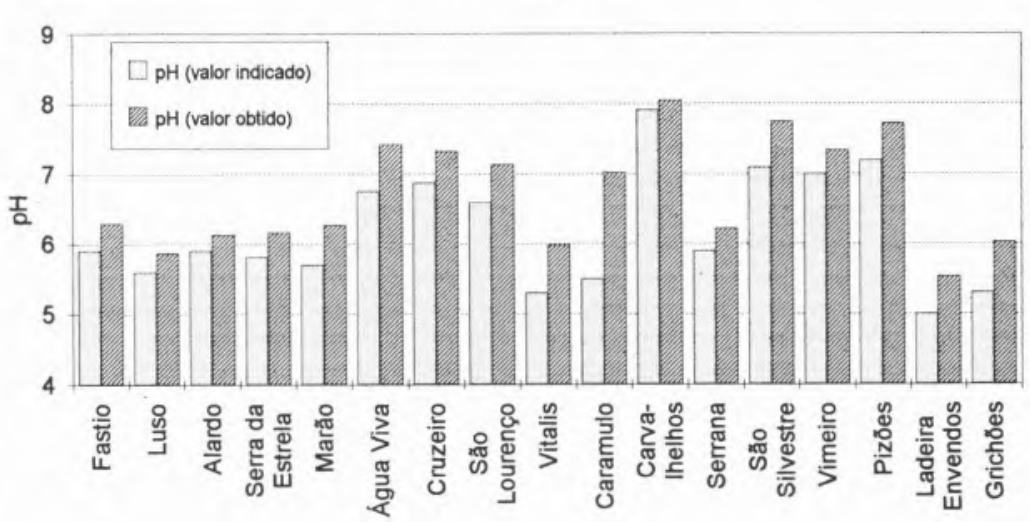

Fig. 2 - Comparação entre os valores de resíduo seco indicados nos rótulos e os obtidos.

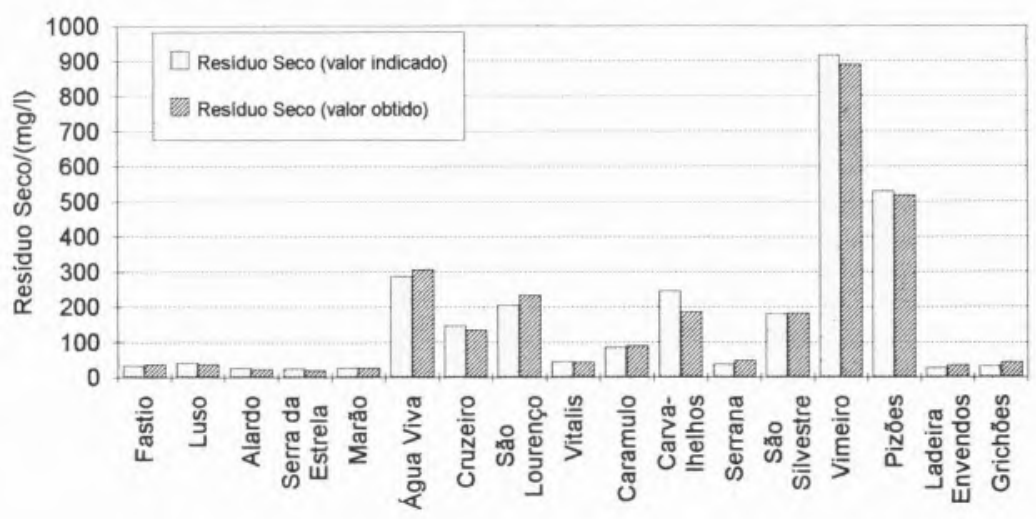

Fig. 3 - Comparaçăo entre os valores de sílica indicados nos rótulos e os valores de sílica molibdoreactiva obtidos.

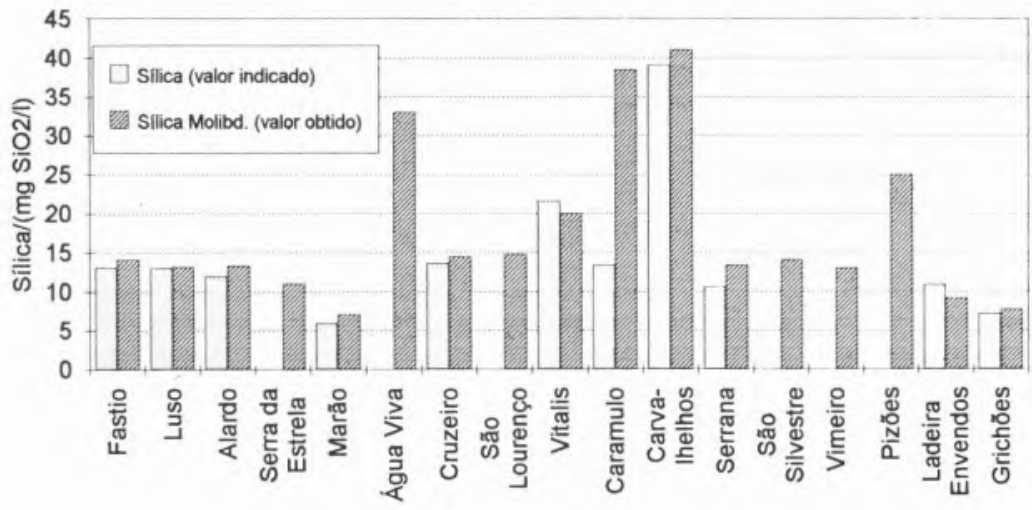

Fig. 4 - Comparação entre os valores de concentração de hidrogenocarbonato (bicarbonato) indicados nos rótulos e os obtidos.

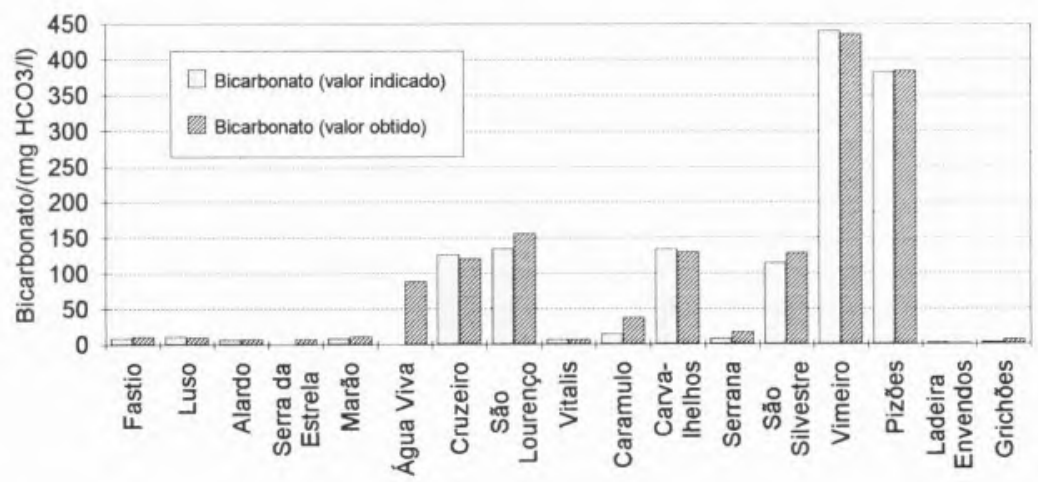




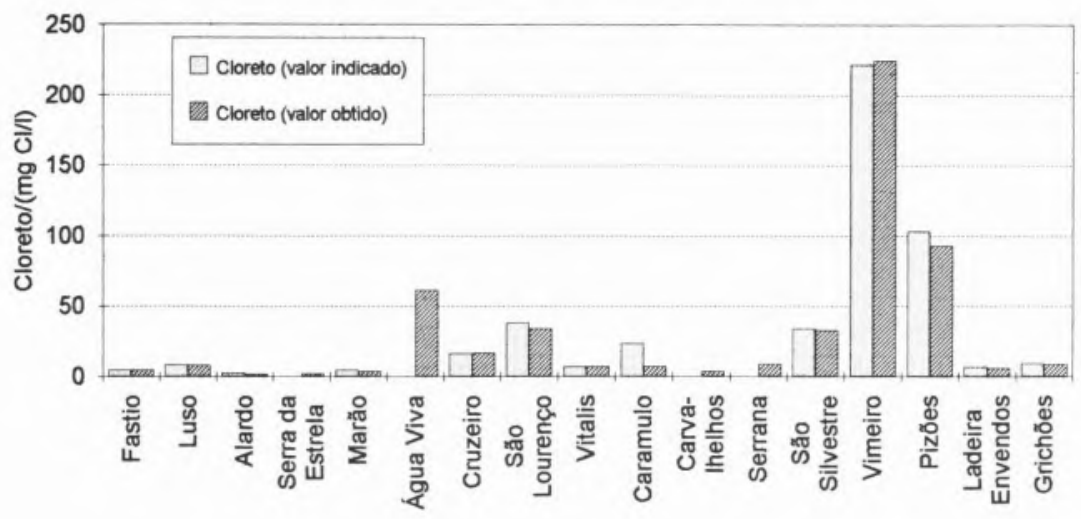

Fig. 6 - Comparação entre os valores da concentraçăo de sulfato indicados nos rótulos e os obtidos.

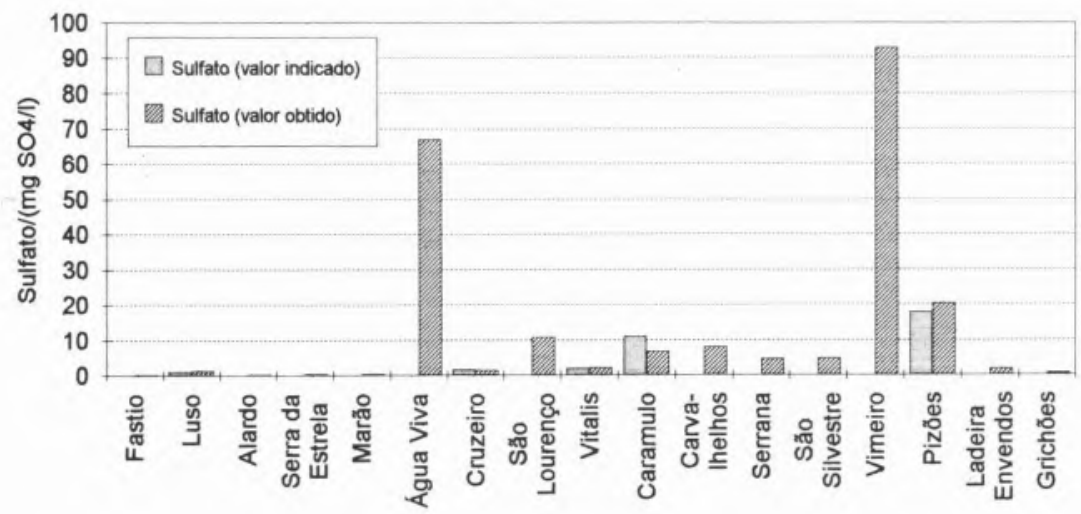

Fig. 7 - Comparaçăo entre os valores da concentração de cálcio indicados nos rótulos e os obtidos.

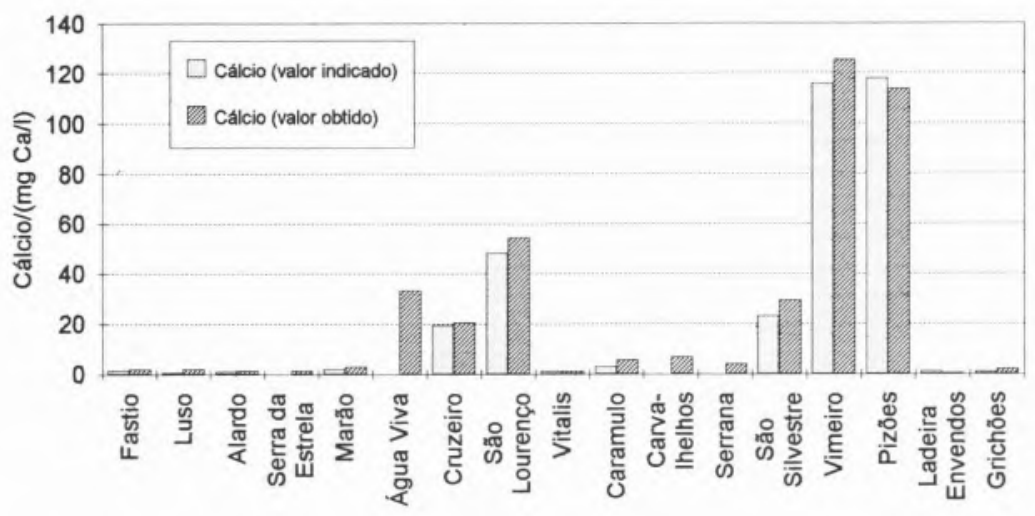

Fig. 8. - Valores obtidos de dureza total, dureza cálcica e dureza magnésica para as 17 águas analisadas.

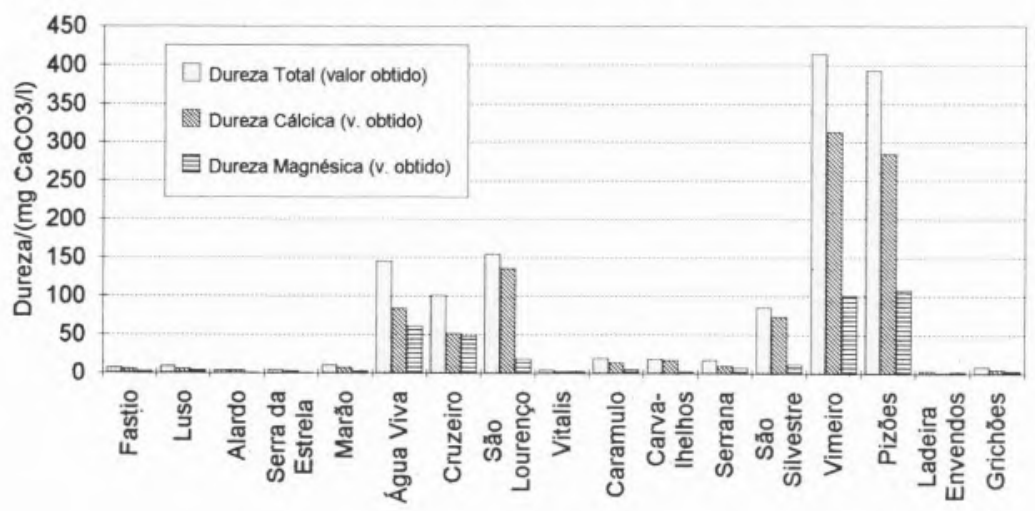

Lourenço", "S. Silvestre", "Vimeiro" e "Pizões".

Da Fig. 6 conclui-se que poucas águas indicam a concentração em sulfato, não havendo grandes diferenças entre os valores obtidos e os indicados (quando 0 são). Apenas as águas "Vimeiro" e "Água Viva" ultrapassam o VMR de $25 \mathrm{mg} \mathrm{SO}_{4} 2-1 /$.

A Fig. 7 mostra-nos as concentrações em cálcio, verificando-se uma razoável concordância entre os valores obtidos e os indicados; apenas duas águas ultrapassam o VMR de $100 \mathrm{mg}$ $\mathrm{Ca}^{2+} / /$ ("Vimeiro" e "Pizões").

A Fig. 8 apresenta as durezas obtidas para as águas analisadas em termos de dureza total e das suas $2 \mathrm{com}$ ponentes: dureza cálcica e dureza magnésica. Nenhuma água ultrapassa 0 Valor Máximo Admissivel (VMA), de $500 \mathrm{mg} \mathrm{CaCO} /$ I. De acordo com [4]), uma água com menos de $75 \mathrm{mg}$ $\mathrm{CaCO3/l}$ de dureza total é considerada macia; de 75 a 150 mg CaCO $/$ I considera-se mediamente dura; de 150 a 300 mg $\mathrm{CaCO}_{3}$ / I e considerada dura; a partir dos $300 \mathrm{mg} \mathrm{CaCO} /$ / considera-se muito dura. Segundo este critério temos uma maioria de águas macias, 4 águas médias ("Água Viva", "Cruzeiro", "S. Lourenço" e "S. Silvestre"), e 2 águas muito duras ("Vimeiro" e "Pizões"). No entanto, o VMA fixado prende-se apenas com questões domésticas (prevenção de incrustações nas canalizações), e não com eventuais efeitos menos benefícos na saúde.

A Fig. 9 apresenta uma comparação entre os valores obtidos para o resíduo seco e para a condutibilidade, verificando-se existir uma certa correlação de proporcionalidade directa, como era de esperar, pois a composição química (iónica) das águas é semelhante.

A Fig. 10 apresenta 0 valor da razão entre a condutibilidade e 0 resíduo seco, em função do resíduo $s e c 0$, para as 17 águas analisadas. Daqui se pode concluir haver uma tendência para a razão "condutibilidade/resíduo seco" aumentar à medida que aumentam os valores de resíduo seco. Isto pode ser explicado pelo facto de as águas com maior resíduo seco terem também maiores concentrações em hidrogenocarbonato, ião este que contribui quantitativamente para a condutibilidade, mas apenas em cerca de metade para 0 resíduo seco. Efectivamente, no processo de evaporação e secagem a $180^{\circ} \mathrm{C}$ para a 
Fig. 9 - Comparaçăo entre os valores obtidos de residuo seco e de condutibilidade das águas analisadas.

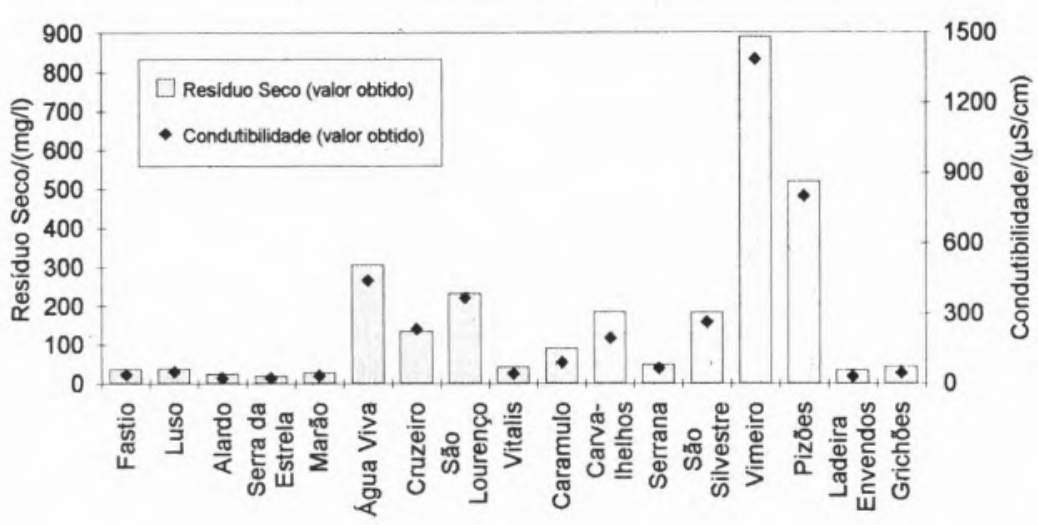

Fig. 10 - Razão entre condutibilidade e residuo seco em função do resíduo seco, para as águas de mesa analisadas.

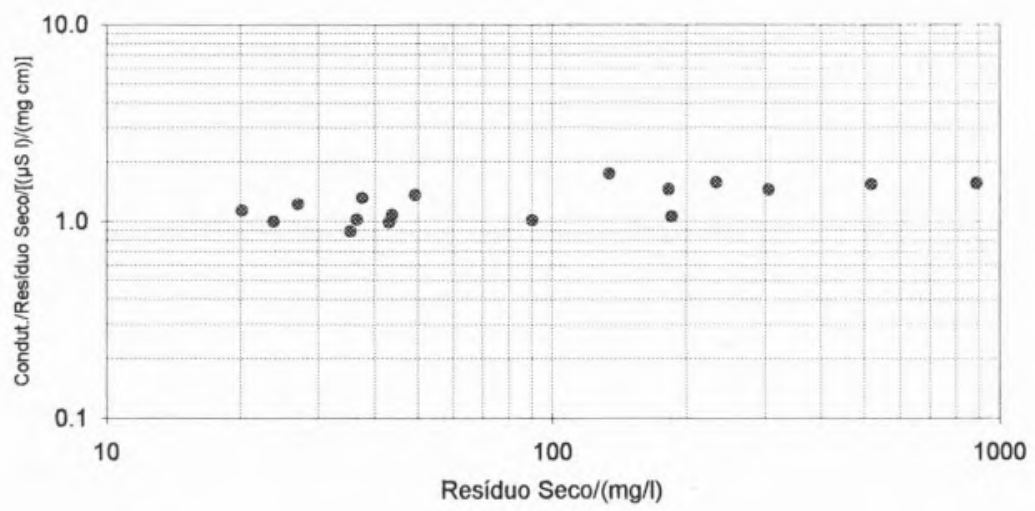

determinação do resíduo seco ocorre a seguinte reaç̧ão:

$$
\begin{gathered}
2 \mathrm{HCO}^{3} \Rightarrow \mathrm{CO}_{2}+\mathrm{CO}_{3}{ }^{2-}+\mathrm{H}_{2} \mathrm{O} \\
(2 \times 61 \mathrm{~g} / \mathrm{mol})^{3}(44 \mathrm{~g} / \mathrm{mol})^{2}(60 \mathrm{~g} / \mathrm{mol})^{2}(18 \mathrm{~g} / \mathrm{mol})
\end{gathered}
$$

Dos produtos de reacção, o dioxido de carbono e a água evaporam, dadas as condições de temperatura, e 0 ião carbonato precipita juntamente com catiões presentes; assim, há uma "perda" teórica de hidrogenocarbonato de $50.8 \%$ $[(2 \times 61-60) /(2 \times 61)]$; experimentalmente, obtivemos perdas de $\mathrm{HCO}_{3}{ }^{*}$ na determinação do resíduo seco de 47.2 a $49.9 \%$, como se pode ver na Tabela 2.

\begin{tabular}{lcc}
\hline $\mathrm{mg} \mathrm{HCO}$ & & \multicolumn{2}{c}{ perda de $\mathrm{HCO}_{3}{ }^{-}(\%)$} \\
\hline 500 & $49.9 ;$ & 49.9 \\
200 & $49.3 ;$ & 49.3 \\
100 & $47.7 ;$ & 48.1 \\
50 & $48.0 ;$ & 47.2 \\
\hline
\end{tabular}

Assim, da Tabela 1 e figuras 1 a 10 tiram-se as seguintes conclusões gerais àcerca das águas de mesa portuguesas analisadas:

1 - Dum modo geral os rótulos indicam muito poucos parâmetros relativamente aos que deviam indicar para caracterizar a água [desde 2 parâmetros ( $\mathrm{pH}$ e Resíduo Seco) até um máximo de 12, sendo 0 número médio de 7-8 parâmetros]. As nossas análises são, assim, muito mais completas (16 parâmetros analisados).

2 - Geralmente, os valores indicados nos rótulos coincidem razoavelmente com as nossas análises, o que leva a crer corresponderem à realidade. Há que ter em consideraçao o intervalo de tempo entre as análises indicadas no rótulo e as análises por nos efectuadas. Há um intervalo que vai de cerca de 1 ano ("Grichões", "Vitalis" e "Serra da Estrela") até 6 anos ("Serrana") entre a data indicada no rótulo e a data das nossas análises. Exceptua-se um caso ("Caramulo") em que apesar do Resíduo Seco ser da mesma ordem de grandeza, os parâmetros mais significativos (tais como pH, Sílica, Bicarbonato e Cloreto) estao muito distantes dos indicados no rótulo (valores estes que se repetiram numa $2^{\mathrm{a}}$ análise de diferente amostra da mesma água).

É evidente que é natural que na maioria dos casos a data indicada no rótulo seja anterior a data daquele específico engarrafamento e, por isso, embora em princípio próxima, não se aplique àquela especiifica amostra.

3 - De um modo geral a composição das águas situa-se dentro dos limites estipulados pelo Decreto-Lei $n^{\circ} 74 / 90$ de 7 de Março para águas de abastecimento para consumo humano, havendo apenas as excepções atrás citadas.

\section{REFERÊNCIAS}

1. L.S. Clesceri, A.E. Greenberg and R.R. Trussel, Standard Methods for the Examination of Water and Wastewater, APHA-AWWA-WPCF, 17th ed. (1989).

2. Jean Rodier, L'Analyse de l'Eau, eaux naturelles, eaux résiduaires, eau de Mer, $7 \mathrm{e}$ ed. Dunod, Paris (1984).

3. Supomos que foi este facto que deu origem a comentários na imprensa segundo os quais 0 Decreto-Lei 74/90 seria tão rigoroso que nem a "Água de Luso" seria por ele "aprovada"!.

4. Russel E. Train, Quality Criteria for Water, U.S. Environmental Protection Agency, Washington D.C., Castle House Publications Ltd (1979).

\footnotetext{
*Departamento de Química - Universidade de Coimbra

** Universidade Lusiada - Vila Nova de Famalicão
} 


\section{GERB $R$ INSTRUM NTS}

(TMT) TAMSON

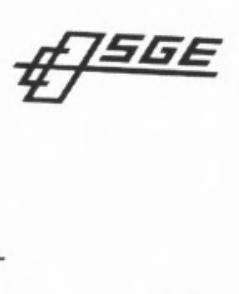

fispers Fishersentific

KOJAIR

\section{METTLER TOLEDO}
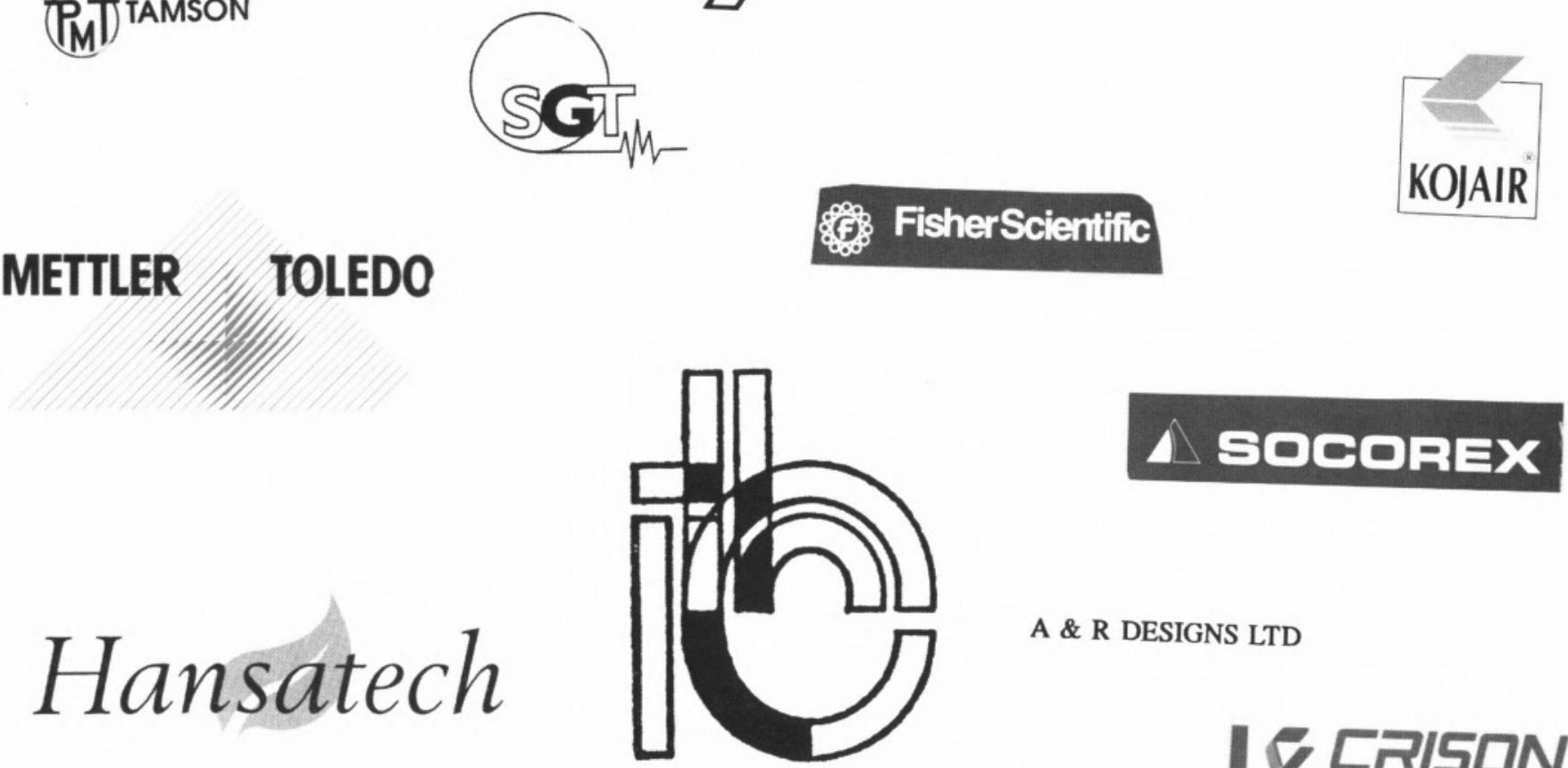

A \& R DESIGNS LTD

\section{Allitech}

UNIEQUIP
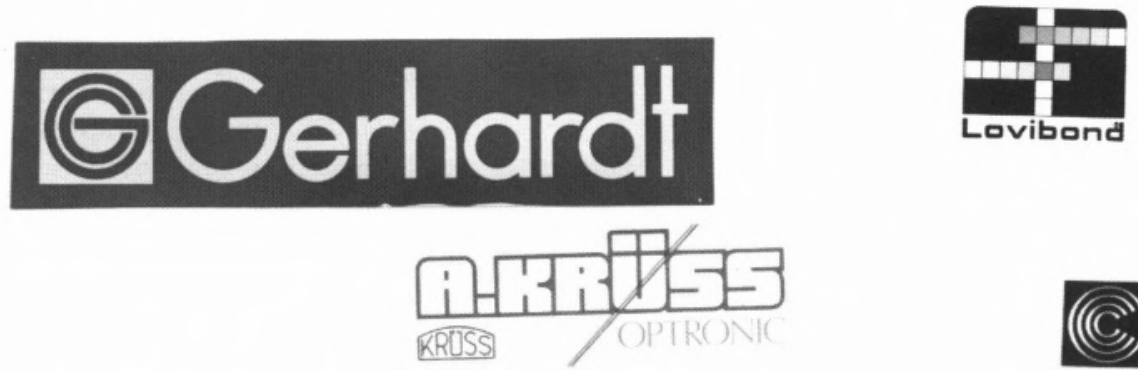

Cathodeon

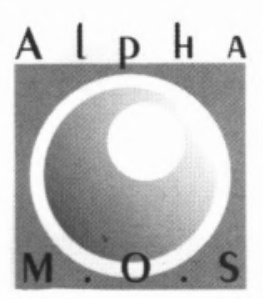

Promochem

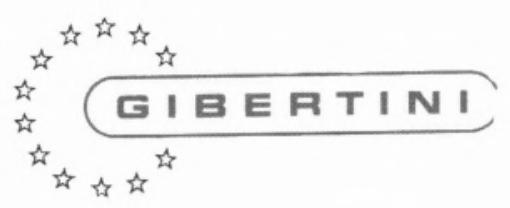

\section{CONSOIRT}

